\title{
Missing Link Found? - The "runaway" path to supermassive black holes
}

\author{
Toshikazu Ebisuzaki \\ Advanced Computer Center, RIKEN , 2-1 Hirosawa, Wako 351-0198, \\ Japan
}

\begin{abstract}
Observations of stellar kinematics, gas dynamics and masers around galactic nuclei have now firmly established that many galaxies host central supermassive black holes (SMBHs) with masses in the range $10^{6} \sim 10^{9} \mathrm{M}_{\odot}$. However, how these SMBHs formed is not well understood. One reason for this situation is the lack of observations of intermediatemass BHs (IMBHs), which could bridge the gap between stellar-mass BHs and SMBHs. Recently, this missing link (i.e., an IMBH) has been found in observations made by ASCA and Chandra of the central region of the starburst galaxy M82 (Matsumoto and Tsuru 1999, Ptak and Griffith 1999, Matsumoto et al. 2001, Kaaret et al. 2001). Subsequent observations by SUBARU have revealed that this IMBH apparently coincides with a young compact star cluster. Based on these findings, we propose a new formation scenario for SMBHs. In this scenario, IMBHs first form in young compact star clusters through runaway merging of massive stars. While these IMBHs are forming, the host star clusters sink toward the galactic nucleus through dynamical friction, and upon evaporation deposit their IMBHs near the galactic center. The IMBHs then form binaries and eventually merge via gravitational radiation, forming an SMBH.
\end{abstract}

\section{Introduction}

There is rapidly growing evidence for SMBHs in the centers of many galaxies [for a review, see Kormendy and Richstone (1995)]. There are too many examples to list here; indeed, although relatively few galaxies show conclusive evidences for central black holes, even fewer galaxies exist for which observations indicate that a central SMBH does not exist (Kormendy and McClure 1993).

Many authors have pointed out that the mass of the central black hole $m_{B H}$ correlates with the mass of bulge $M_{b}$, i.e., the ratio of $m_{B H}$ to $M_{b}$ is almost constant at 0.002 (Kormendy and Richstone 1995); 0.006 (Magorrian et al. 1998); 0.001 (Merritt and Ferrarese 2001). This suggests that the formation of the central $\mathrm{BH}$ is somehow related to that of the bulge.

Our theoretical understanding of the formation mechanism of SMBHs has not advanced much beyond the scenarios described by Rees $(1978,1984)$ in the early 1980s. In the famous diagram by Rees, there were basically two paths from gas clouds to supermassive black holes. The first is direct monolithic collapse. 
The second is via the formation of a star cluster, with subsequent runaway collisions leading to $\mathrm{BH}$ formation. Previous numerical studies, however, have demonstrated that neither path is likely. In the first, a massive gas cloud is much more likely to fragment into many small clumps, in which stars then form. Therefore, direct formation of a massive $\mathrm{BH}$ from a gas cloud seems difficult. In the second, stellar dynamics in star clusters does not easily lead to the formation of supermassive black holes. A number of low-mass black holes (masses around $10 \mathrm{M}_{\odot}$ ) are formed via the evolution of massive stars, and these black holes do indeed sink to the center of the cluster through dynamical friction and form binaries by three-body encounters. Taniguchi et al. (2000) argued that IMBHs could be formed through successive merging of compact objects. However, recent $N$-body simulations (Portegies Zwart and McMillan 2000) have demonstrated that practically all of these black-hole binaries are ejected from the cluster by recoil of interactions with other black holes (or BH binaries) before they merge through gravitational radiation.

\section{IMBHs in $\mathbf{M 8 2}$}

Matsumoto et al. (2001) have identified nine bright compact X-ray sources in the central region of M82 using recent Chandra data. The brightest source (No. 7 in their Table 1) had a luminosity of $9 \times 10^{40} \mathrm{ergs}^{-1}$ in Jan 2000, corresponding to a black hole with a minimum mass of $700 \mathrm{M}_{\odot}$ (assuming the Eddington luminosity). It probably consists of a single compact object, as its X-ray flux shows rapid time variation (Matsumoto et al. 2001). This is the first detection of a $\mathrm{BH}$ candidate with a mass much greater than $100 \mathrm{M}_{\odot}$ but much less than $10^{6} \mathrm{M}_{\odot}$. Among the eight other sources, at least three $(5,8$ and 9$)$ have Eddington masses greater than $30 \mathrm{M}_{\odot}$.

Matsushita et al. (2000) observed the same region with Nobeyama Millimeter Array and found a huge expanding shell of the molecular gas. They estimated the age and kinetic energy of the shell to be around $1 \mathrm{Myr}$ and $10^{55}$ erg, suggesting that a strong starburst took place a few Myr ago.

Harashima et al. (2002) observed the same region in the IR (J, H, and $\mathrm{K}^{\prime}$ band) using the CISCO instrument on the SUBARU telescope. They identified a number of young compact star clusters, at least four of them coinciding with the X-ray sources within the positional uncertainties of Chandra and SUBARU. The other five Chandra X-ray sources are far outside the central starburst region of M82. Even so, two of them coincide with infrared sources in the 2MASS point source catalog. The logical conclusion from these observations is that most of the Chandra X-ray sources, including the brightest one with an Eddington mass of $700 \mathrm{M}_{\odot}$, were formed in star clusters.

Therefore, we now have two important observational results. The first is that a $\mathrm{BH}$ with intermediate mass $\left(100<M_{b h} / M_{\odot}<10^{6}\right)$ may have been found. The second is that it coincides with a young compact star cluster. In the following, we discuss how these findings change our understanding of the formation of supermassive BHs. We first discuss how IMBHs can be formed in young compact star clusters, then how IMBHs might grow into SMBHs. 


\section{IMBH formation through runaway growth}

What the observations suggest is that IMBHs are formed in young compact clusters. Here, again, we have essentially two possible paths, as we described in the introduction: Direct collapse of gas cloud and runaway collision in the star cluster. The first path is still difficult, but the situation is quite different with the second path.

Previous studies of runaway merging mainly consider old globular clusters, and therefore the mass of stars were pretty small. In fact, most massive stars are stellar-mass black holes or neutron stars, which are quite difficult to merge together. In young clusters, however, massive stars are still there, and the collisions and mergings are much easier to take place.

In our proposed scenario, IMBHs form and grow through successive merging of massive stars (and IMBHs) in dense star clusters (see figure 1). More massive stars in star clusters have higher merging rates than less massive cluster members (or field stars) because of their larger geometrical cross sections and a stronger gravitational focusing, and concentration to the central region by mass segregation in the cluster. In addition, complex resonances in binary-single star encounters contribute to a significant increase in merging rate of massive stars (Hut and Inagaki 1985, McMillan 1986). If these effects are strong enough, we expect that a "merging instability" (Lee 1987), or a runaway growth of the most massive star, will occur in the cluster core. In fact, $N$-body simulations carried out by Portegies Zwart et al. (1999) have demonstrated that runaway merging can take place in a systems containing only $\sim 12,000$ stars, before stellar evolution eliminates the most massive stars.

Portegies Zwart et al. found that, in one case, the most massive star experienced more than ten collisions and reached a mass of around $200 \mathrm{M}_{\odot}$ before evolving into a supernova. There is considerable uncertainty as to how much mass would remain as a $\mathrm{BH}$ after the supernova explosion of such a massive star, but it is quite likely that the remnant black hole would still be one of the most massive objects in the cluster, and that the runaway merging process would continue. Although the geometrical cross section of a $\mathrm{BH}$ is small, "merging" would take place when a star approached within its tidal radius, leading to a relatively large merger cross section.

In order for runaway merging to occur, dynamical friction timescale for the most massive stars must be short enough that they can sink to the center during their lifetime of several Myr. The dynamical friction timescale may be expressed as follows [equation 7-26 in Binney and Tremaine 1987]:

$$
t_{\text {fric }}=\frac{1.17}{\log \Lambda} \frac{r^{2} v_{c}}{G m} \simeq 2.7 \times 10^{7}\left(\frac{r}{1 \mathrm{pc}}\right)^{2}\left(\frac{r_{h}}{10 \mathrm{pc}}\right)^{-1 / 2}\left(\frac{M}{10^{6} M_{\odot}}\right)^{1 / 2}\left(\frac{20 M_{\odot}}{m}\right) \mathrm{yr}
$$

where $\log \Lambda$ is the Coulomb logarithm, $G$ is the gravitational constant, $v_{c}$ is the local velocity dispersion, $r$ is the distance from the center of the cluster, $r_{h}$ and $M$ are the half-mass radius and the total mass of the cluster, and $m$ is the mass of the star. Here, it is assumed that the background stellar distribution is that of the singular isothermal sphere. Thus, if the cluster has a very large core, the above equation underestimates the timescale for stars in the core. Such a large core, however, is probably unlikely. 
In the following, we consider how dynamical friction works in the cluster found in M82. From the infrared luminosity, Harashima et al. (2002) estimate that the total mass of the cluster is $\sim 5 \times 10^{6} \mathrm{M}_{\odot}$. They also estimated the seeingcorrected radius of the cluster as $5 \mathrm{pc}$, which is most likely a good estimate of $r_{h}$. For $r=0.5 \mathrm{pc} \sim 0.1 r_{h}$, a volume which contains about $5 \%$ of the total cluster mass, the dynamical friction timescale is less than $10 \mathrm{Myr}$. We therefore conclude that a significant fraction of the most massive stars sink to the cluster center and undergo runaway merging before exploding as supernovae. Note that the radius at which the dynamical friction timescale is short enough is relatively large. Therefore, unless the cluster had initially a very large core whose size is more than $10 \%$ of the half-mass radius, the condition for runaway merging is satisfied.

After the $\mathrm{BH}$ has become much more massive than other cluster members, it forms a cusp near the cluster center (Bahcall and Wolf 1976), and continues to swallow other stars. Unfortunately, no realistic simulations of this phase of the evolution are available. Marchant and Shapiro (1980) performed Monte-Carlo simulations of this stage for a simplified cluster containing $3 \times 10^{5}$ solar-mass stars and one 50 solar-mass seed $\mathrm{BH}$. They found that the $\mathrm{BH}$ mass jumped to over $10^{3} M_{\odot}(0.3 \%$ of the cluster mass) almost immediately after they put the $\mathrm{BH}$ into the system. After this initial rapid growth, a slower phase ensued, with a doubling timescale comparable to the relaxation time of the cluster. Their result should be regarded as a lower limit on the $\mathrm{BH}$ growth rate, since realistic effects, in particular the presence of a mass spectrum, would greatly enhance the accretion rate. Taking these effects into account, it seems safe (even conservative) to suppose that $0.1 \%$ of the total cluster mass accretes to form $\mathrm{a} \sim 5000 M_{\odot}$ central $\mathrm{BH}$ in a few Myr.

As stated above, there are more than 10 bright star clusters in the vicinity of the IMBH host cluster in M82, some of them apparently hosting small BHs. Their age is around $10 \mathrm{Myr}$ (Harashima et al. 2002). Also, the starburst in M82 is a long-duration event, having started at least $200 \mathrm{Myr}$ ago (de Grijs et al. 2001). If we assume that clusters form at a constant rate, we conclude that around 200 clusters have been formed. We believe it is safe to assume that around one hundred clusters similar to our host cluster have formed in total, and that a considerable fraction of them host IMBHs.

\section{Building up the central SMBH}

We now describe how IMBHs formed in star clusters combine to form a central $\mathrm{SMBH}$ (see also figure 2). The growth rate of the IMBH in a star cluster slows down once all the massive stars are swallowed (after $\sim 100 \mathrm{Myr}$ ). Subsequently the cluster is subject to two evolutionary processes: evaporation through twobody relaxation and orbital decay (sinking) via dynamical friction. Evaporation is driven partly by thermal relaxation and partly by stellar mass loss. Portegies Zwart et al. (2001a, 2001b) estimated that the evaporation timescale for a tidally limited compact star cluster is around 2-3 half-mass relaxation times, which is of the order of a few Gyr for our star clusters. Rewriting equation (1) using appropriate scaling for this case, we find that the timescale on which the cluster 


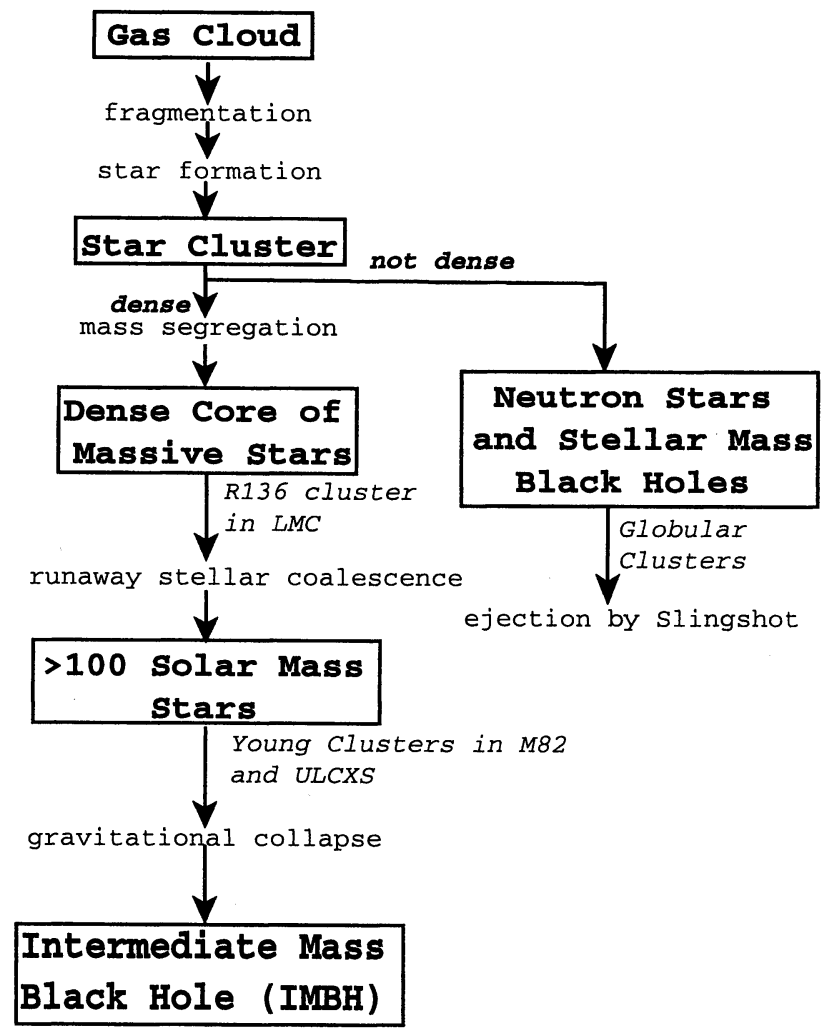

Figure 1. Schematic diagram of the formation process of an intermediate-mass black hole (IMBH). A gas cloud fragments to form many less massive clouds as it cools by radiation. Many stars are formed through this fragmentation, and a star cluster comes into being. There are two possible evolutionary paths for this cluster, depending on its stellar density. If the star cluster is so dense that stellar mass segregation is faster than stellar evolution for the most massive stars (time scale $\sim 10^{6} \mathrm{yr}$ ), those stars sink to the cluster core by dynamical friction, and form a dense inner core of massive stars at the cluster center. In this inner core, the massive stars undergo a runaway stellar merging and a very massive star with mass exceeding $100 M_{\odot}$ forms. This very massive star eventually collapses into a black hole, which continues to grow by swallowing nearby massive stars. If the cluster is not dense enough for mass segregation to occur in $10 \mathrm{Myr}$, massive stars evolve into compact stellar remnants such as neutron stars and stellar-mass black holes $\left(\sim 10 M_{\odot}\right)$. Those stellar remnants slowly sink to the cluster center, since they are heavier than other stars in the system, and eventually form binaries. Successive three-body interactions make these binaries more tightly bound, and eventually they are ejected from the cluster by the slingshot mechanism. 
sinks to the galactic center via dynamical friction is

$$
t_{\text {fric }} \simeq 6 \times 10^{8}\left(\frac{r}{1 \mathrm{kpc}}\right)^{2}\left(\frac{v_{c}}{100 \mathrm{kms}^{-1}}\right)\left(\frac{5 \times 10^{6} M_{\odot}}{m}\right) \mathrm{yr} .
$$

Clusters initially within $1 \mathrm{kpc}$ of the galactic center can therefore reach the center within one Gyr. Note that an IMBH can reach the galactic center only if its host cluster can sink to the center before it evaporates. If the cluster dissolves before significant orbital decay occurs, the timescale for the IMBH to fall to the center increases greatly.

According to our estimate in the previous section, around 100 compact clusters have formed close to the center of M82 in the last 200 Myrs. If we assume that half of these clusters contain $5000 \mathrm{M}_{\odot} \mathrm{IMBHs}$, and that these IMBHs actually merge, then the total $\mathrm{BH}$ mass at the center of the galaxy will be at least $2.5 \times 10^{5} \mathrm{M}_{\odot}$.

Having demonstrated that $5000 \mathrm{M}_{\odot}$ IMBHs can form and reach the galactic center in a reasonable timescale, we now turn to the question of whether the multiple IMBHs at the center can merge. Begelman et al. (1980) discussed the evolution of a supermassive $\mathrm{BH}$ binary at the center of a galaxy, taking into account dynamical friction from field stars and energy loss via gravitational radiation. They found that the merging timescale depends strongly on mass, and for a very massive $\mathrm{BH}$ with a mass of $10^{8} \mathrm{M}_{\odot}$ in which they were interested, merging took much longer than a Hubble time.

For the IMBHs, however, the timescale for merging through gravitational radiation is many orders of magnitude shorter than that for the SMBHs considered by Begelman et al. (1980), because the loss-cone depletion is not as effective as Begelman et al. assumed, at least for relatively small BH mass. The loss-cone is filled in the timescale related to the central relaxation time of the cluster, which is much shorter than a cluster with IMBHs than that for a galaxy with SMBHs. Thus, IMBHs can reach high orbital velocities in short timescales. Recent extensive numerical simulations (Makino et al. 1993, Makino 1997, Quinlan and Hernquist 1997) have shown that the hardening of the $\mathrm{BH}$ binary through dynamical friction is in fact several orders of magnitude faster than the prediction from loss-cone arguments. Though the number of particles employed (up to 256k) was not large enough to model SMBH binaries, it was certainly large enough to model evolution of IMBH binaries. Based on the N-body simulations above, Merritt (1999) estimated the timescale of merging (first through dynamical effects and then through gravitational radiation) as $T \sim 1.4 \times 10^{5}\left(\mathrm{M} / 10^{4} \mathrm{M}_{\odot}\right)\left(\sigma / 200 \mathrm{kms}^{-1}\right)^{-4}$. We can safely conclude that the merging time scale for IMBHs with masses less than $10^{4} \mathrm{M}_{\odot}$ is one Myr or less.

Once one $\mathrm{BH}$ has become more massive than typical infalling $\mathrm{BHs}$, it becomes extremely unlikely that it will be ejected, since the recoil velocity from three-body interactions is inversely proportional to the mass (because of momentum conservation). Thus, even though some of the infalling BHs might be ejected by the slingshot mechanism, the central $\mathrm{BH}$ will continue to grow. 


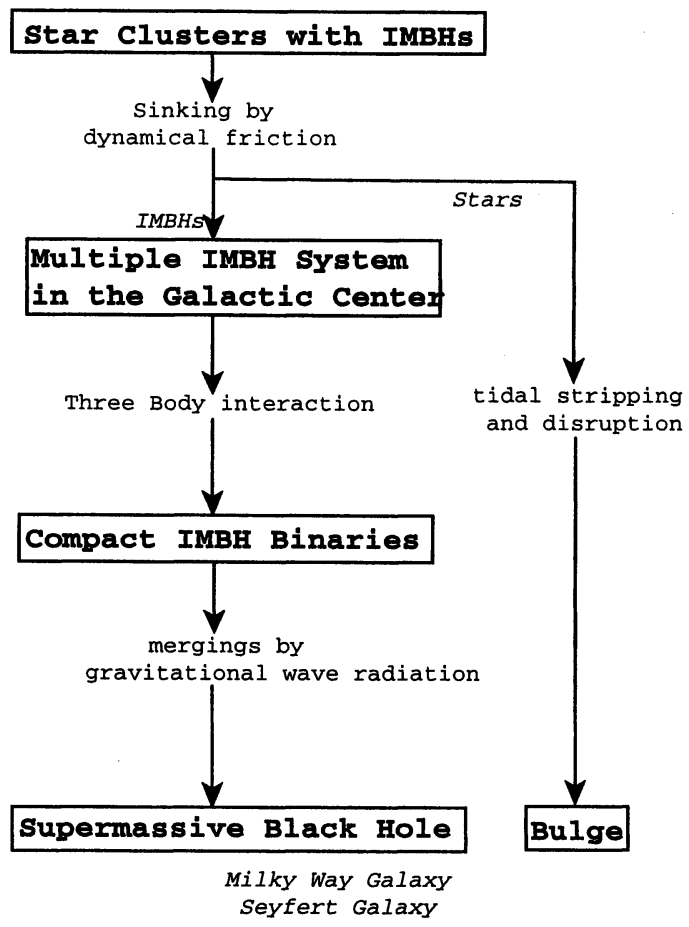

Figure 2. Schematic diagram of the formation of supermassive black holes from star clusters containing intermediate-mass black holes (IMBHs). The star clusters sink to the galactic center by dynamical friction. The tidal field of the parent galaxy strips stars from the outskirts of the cluster. Those stripped stars ultimately become part of the galactic bulge. The IMBHs carried to the center by the star clusters form a multiple IMBH system at the center of the galaxy. IMBH binaries are formed and become harder and harder by three-body interactions with other IMBHs. Eventually, they merge into one or more massive black holes through gravitational radiation. Successive mergings of IMBHs form a supermassive black hole with a mass of $\sim 10^{6} \mathrm{M}_{\odot}$ 


\section{Discussion}

In this paper, we have discussed the implications for our understanding of the SMBH formation mechanism of the recent discovery of an IMBH in M82. Our conclusion is that the IMBH found in M82 plays the role of "missing link" between stellar-mass BHs and SMBHs.

Since we now have the first candidate for IMBHs, it seems natural to expect that SMBHs might be formed from them. We propose that IMBHs are formed in the cores of young compact star clusters through mergings of massive stars and $\mathrm{BHs}$ formed from them. These compact young clusters sink to the galactic center by dynamical friction. At the same time, they evaporate via thermal relaxation, stellar mass loss and the effect of the parent galaxy's tidal field. Thus, IMBHs are created and transported to the center of the galaxy, where they eventually merge to form SMBHs.

In the following, we discuss how we might confirm our new scenario. The most direct evidence would be the observation of gravitational radiation from close binary IMBHs or merging IMBHs. LISA (Jafry, Cornelisse, and Reinhard 1994), when completed, will be able to detect IMBH merging events even at cosmological distances. The formation rate of SMBH is estimated to be one per $1 \sim 10$ years. In our scenario, each SMBH is a product of $\sim 100$ mergings of IMBHs or IMBH and growing SMBH. Therefore we predict a much higher event rate for IMBH-IMBH and IMBH-SMBH merging, of the order of 1 per month or even 1 per week.

To test our hypothesis, searches for IMBHs in other galaxies are clearly necessary. In our view, IMBHs are likely to form in young compact star clusters created in nuclear starbursts. We predict that coordinated observations of nearby starburst galaxies at IR, X-ray and radio wavelengths, like those performed for M82, will reveal many more candidate IMBHs. In particular, the Ultra Luminous Compact X-ray Sources (Makishima et al. 2000, Colbert and Mushotzky 1999) may be directly related to IMBHs.

It is also vital to determine internal and external kinematics of the host star clusters of IMBHs. High-dispersion spectroscopy in the IR with large groundbased telescopes such as SUBARU should be able to determine the velocity dispersion of such a star cluster. Observations by HST would resolve the cluster and give us detailed information of its structure. Comparison of these results with theoretical models will then determine whether or not runaway merging can actually take place there.

The explosive star formation, induced by interactions or collisions of galaxies, are much more frequent in the earlier $(Z \sim 5)$ phase of the universe. The formation of supermassive black holes by the scenario presented here could also explain the peak in the distribution of quasars at $z=2 \sim 5$.

\section{References}

Bahcall, J. N., and Wolf, R. A., 1976, ApJ, 209, 21

Begelman, M. C., Blandford, R. D., and Rees, M. J., 1980, Nature, 287, 307 
Binney J. and Tremaine S., 1987, Galactic Dynamics, Princeton University Press, Princeton.

Colbert, E.J.M. and Mushotzky, R.F., 1999, ApJ, 519, 89

de Grijs, R., O’Connell, R. W., and Gallagher, J. S., 2001, AJ, 121, 768

Harashima, T., et al., 2002, in preparation

Hut, P. and Inagaki, S., 1985, ApJ, 298, 502

Jafry Y.R., Cornelisse J., and Reinhard R., 1994, LISA - a laser interferometer space antenna for gravitational-wave measurements. ESA Journal 18: 219-228

Kaaret, P., Prestwich, A.H., Zezas, A., Murray, S.S., Kim, D.W., Kilgard, R.E., Schlegel, E.M., and Ward, M.J., 2001, MNRAS, 321, L29

Kormendy, J. and McClure, R.D., 1993, AJ, 105, 1793

Kormendy, J. and Richstone, D., 1995, ARA\&A, 33, 581

Lee, H.M., 1987, ApJ, 319, 801

Magorrian et al., 1998, AJ, 115, 2285

Makino, J., Fukushige, T., Okumura, S.K., and Ebisuzaki, T., 1993, PASJ, 45, 303

Makino, J., 1997, ApJ, 478, 58

Makishima, K. et al. 2000, ApJ, 535, 632

Matsumoto, H. and Tsuru, T., 1999, PASJ, 51, 321

Matsumoto, H., Tsuru, T., Koyama, K., Awaki, H., Canizares, C.R., Kawai, N., Matsushita, S., and Kawabe, R. 2001, ApJ, 547, L25

Matsushita, S., Kawabe, R., Matsumoto, H., Tsuru, T. G., Kohno, K., Morita, K., Okumura, S.K., and Vila-Vilar, B. 2000, ApJ, 545, L107

Marchant, A. B. and Shapiro, S. L., 1980, ApJ, 239, 685

McMillan, S. L. W., 1986, ApJ, 306, 552.

Merritt, D. to appear in "Galaxy Dynamics: From the Early Universe to the Present", eds. F. Combes, G. Mamon and V. Charmandaris.

Merritt, D. and Ferrarese, L., 2001, MNRAS, 320, L30.

Portegies Zwart, S.F., Makino, J., McMillan, S. L. W., and Hut, P., 1999, A\&A, 348, 117

Portegies Zwart, S.F., and McMillan, S. L. W., 2000, ApJ, 528, L17 
Portegies Zwart, S.F., Makino, J., McMillan, S. L. W., and Hut, P., 2001a, ApJ, 546, L101

Portegies Zwart, S.F. Makino, J. McMillan, S.L.W., and Hut, P., 2001b, ApJ in press.

Ptak, A. and Griffith, R., 1999, ApJ, 535, L85

Quinlan, G.D. and Hernquist, L., 1997, New Astronomy, 2, 533.

Rees, M. J., 1978, The Observatory, 98, 210.

Rees, M. J., 1984, ARA\&A, 22, 471.

Taniguchi, Y., Shioya, Y., Tsuru, T.G., Ikeuchi, S., 2000, PASJ, 52, 533 